\title{
Development and Validation of a Numerical Model for Vibration of Power Lines
}

\author{
Mohammad Hadi Jalali \\ Faculty of Engineering and Applied Science \\ Memorial University \\ St. John's, Canada \\ mjalalinodou@mun.ca
}

\author{
Geoff Rideout \\ Faculty of Engineering and Applied Science \\ Memorial University \\ St. John's, Canada \\ gdrideout@mun.ca
}

\begin{abstract}
Modal testing is being investigated as a means of non-destructive evaluation of wooden utility pole strength. In order to understand the effects of conductors on the dynamics of the poles, a numerically efficient model based on lumped segments for the conductor has been developed and experimentally validated. The cable is modeled as number of lumped segments jointed with axial and torsional springs and dampers representing the cable's compliance and damping. In order to validate the models, an experimental set up for vibration testing of the cable has been built. Time response measurement and modal testing are performed and the comparison of the experimental results with the numerical results show that the lumped segment model has the fidelity to capture the dynamics of the cables efficiently and accurately.
\end{abstract}

Keywords; Cable dynamics; Lumped segment model; Modal testing; Bond graph model

\section{INTRODUCTION}

Wooden utility poles are extensively used in North America for electrical power transmission and distribution. These wooden poles are subject to deterioration due to aging, rot, woodpecker damage, and fungal attack. Developing a nondestructive pole evaluation method based on modal testing requires numerical modeling of the poles and attached cables (conductors). Because cables are attached to the utility poles, vibrations of the cables affect the measurement of the modal properties of the poles. Therefore, a numerically efficient model should be developed to capture the dynamics of the cables.

Barry et. al. [1] investigated the vibrations of a single transmission line conductor with attached Stockbridge damper. They modeled the system as a double-beam system and derived the equations of motion of the system using Hamilton's principle. They validated the analytical results with experimental results and investigated the effect of damper location and characteristics on the conductor natural frequencies. Ricciardi et al. [2] developed a continuous model for vibration analysis of cables with sag considering the bending stiffness. They derived the vibration equation of motion of a sagged cable as an Euler-Bernoulli beam and solved the equation by an exact method. They performed a parametric study to investigate the effect of sag and bending stiffness on the cable natural frequencies and validated the results using finite element (FE) and finite difference methods. The exact method that they used could not be used when the cable is attached to a pole or moving support structure. Barbieri et. al. [3] used linear and nonlinear finite element models for analyzing the dynamic behavior of three different transmission line cables. They validated the numerical results by experimental tests and investigated the effect of inclusion of Stockbridge damper and linearity of the FE model on the natural frequencies. They concluded that linear finite element models provide good results for short cables only. Li et. al [4] and Wang et. al [5] presented a simplified computational model of a high-voltage transmission tower-line system under out-ofplane and in-plane vibrations due to seismic excitations. The transmission cables and their supporting towers were modeled as a lumped mass system. They did not consider the bending stiffness of conductors and their model was a simplified model for obtaining the response to seismic excitations. They obtained the mass and stiffness matrices of the coupled system and derived the linear equations of motion and validated the theoretical results with experiments. Papailiou [6] for the first time proposed a model for conductors that took into account the interlayer friction and the interlayer slip in the conductor during bending, obtaining a bending stiffness that changes with the bending displacement and the tension applied to the conductor. Spak et. al. [7] reviewed helical cable models with a focus on cable damping modeling. They described the work done in the literature on the inclusion of damping through frictional effects, variable bending stiffness, and internal friction. Spak et. al. [8, 9] developed the distributed transfer function method to model cables and simple cabled structures. They included shear effects, tension, and hysteretic damping for modeling of helical stranded cables and investigated the effect of cables on the dynamics of cabled structures using the developed model and by experimental tests. Pinto et. al [10] developed a bond graph model for a wooden pole with a cable attached to the free end. In their paper, the cable was modeled as a series of point masses connected by translational springs and the pole was represented 
by a modal expansion based on separation of variables. They obtained the modal parameters of the cable and pole-cable systems numerically and experimentally. They concluded that their model should be expanded and improved from point masses to rigid bodies with rotational inertia, connected with bending stiffness and damping elements as well as axial ones.

The bond graph formalism, details of which can be found in [11] is chosen because of the ease with which it allows expansion of the model by adding segments in a modular way. Bond graphs, which use a small set of generalized elements to model multi-domain systems, facilitate connection of the cable model with other subsystem models such as poles, regardless of whether the poles are modeled using lumped segments or modal expansion.

This paper consists of two main sections. In Section II, a bond graph lumped segment model of the cable is presented. In this model, the cable is modeled as number of segments that are attached to each other by springs and dampers representing the axial and bending compliances and damping of the cable, respectively. Section III describes the experimental set up and procedure that is used for performing time series measurement and modal testing of the stranded cable. The modeling assumptions and the results are explained in Section IV.

\section{LUMPED SEGMENT MODEL}

\section{A. Theoretical Development}

In this paper, the cable is modeled as a pinned-pinned beam divided into number of segments. A numerical solution is obtained by a lumped segment approximation that captures axial and bending motions. To the purpose of modeling, two types of coordinate systems are used in the model. The first coordinate system is the inertial coordinate system. A body-fixed coordinate system is attached to each segment, the origin of which is located at the center of gravity and the local $x$ axis of which is in the axial direction. The advantages of using body coordinate system are that axial springs between the segments can be modeled simply along the local coordinates, and that orientation angles and inertia properties are more easily handled if the model is expanded to three dimensions. Figure 1 shows representative segments of the cable, and local and global coordinate systems.

The cable is represented by a series of rigid cylinders of equal length and cross section; each rigid body has the same properties as the corresponding portion of the cable. As the number of segments approaches infinity, the behavior of the lumped segment model will converge to that of the continuous cable. The length of each segment is defined by the number of rigid bodies, $n$. For each segment, three points are defined: $B$ in the left end of the segment, $A$ in the right end and $G$ in the center of mass. Point $A$ of element $i$ and point $B$ of element $i+1$ are linked by one torsional and two linear springs. The torsional spring represents the bending compliance or flexural rigidity of the beam in the $x-y$ plane, and the linear springs in $x$ and $y$ local directions represent the axial and shear compliance respectively. Each spring is complemented with a damper acting in parallel to it that acts as the cable material damping. Figure 1 shows the connection of each segment to the next and previous segments using torsional and linear springs, also illustrates the body-fixed coordinate system. For simplicity purposes, the dampers are not shown in the figure.

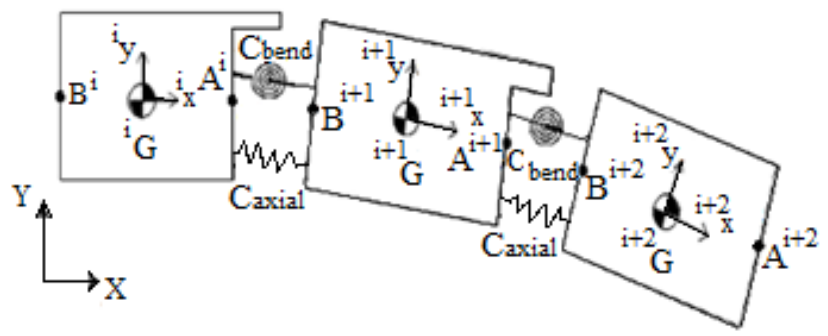

Figure 1. Multibody dynamic model

\section{Cable Compliance:}

Equations (1)-(2) present the axial and bending compliance of the cable, respectively [12].

$$
\begin{aligned}
C_{\text {axial }} & =\frac{E A}{l} \\
C_{\text {bend }} & =\frac{E I}{l}
\end{aligned}
$$

where:

$E=$ Young's modulus of beam

$A=$ Cross sectional area of the cable

$l=$ Length of the cable segment

$I=$ Area moment of inertia of the cross section

\section{Cable Inertia:}

The translational inertia of the lumped segments is equal to their mass. The rotational inertia of a cylinder about the axis perpendicular to the body-fixed coordinate plane, through the center of mass $G$, is as follows.

$$
J_{G}=1 / 12 m\left(3 / 4 d^{2}+l^{2}\right)
$$

Where $m$ is mass of a segment, $d$ is diameter of cable cross section, and $l$ is segment length.

\section{Cable Damping:}

Damping in the cable is due to the material damping associated with the hysteresis energy losses in the material and interlayer wire slip. Because the stranded cable consists of number of layers, the layers can slip on each other during vibration and this interlayer friction increases the damping in the stranded cables. In this paper, axial and bending dampers are placed in parallel with the springs in the model. The damper values are tuned to give decay of the response and natural frequencies close to experimental results. Further work should be done in the future to model the damping in the numerical model more accurately. 


\section{B. Kinematics of Rigid Bodies}

In the bond graph model, we need to develop the velocity relations between points $G$ and $A$ and $B$ in Figure 1. Eq'ns (4)(5) represent the velocity of end points $A$ and $B$ in the local coordinate of the segment, respectively.

$$
\begin{gathered}
{ }^{i} \vec{V}_{A}=\left[\begin{array}{c}
V_{A x} \\
V_{A y}
\end{array}\right]={ }^{i} \vec{V}_{G}+{ }^{i} \vec{V}_{A / G}=\left[\begin{array}{c}
V_{G x} \\
V_{G y}
\end{array}\right]+\left[\begin{array}{c}
0 \\
A G \omega
\end{array}\right] \\
{ }^{i} \vec{V}_{B}={ }^{i}\left[\begin{array}{c}
V_{B x} \\
V_{B y}
\end{array}\right]={ }^{i} \vec{V}_{G}+{ }^{i} \vec{V}_{B / G}={ }^{i}\left[\begin{array}{c}
V_{G x} \\
V_{G y}
\end{array}\right]+{ }^{i}\left[\begin{array}{c}
0 \\
-B G \omega
\end{array}\right]
\end{gathered}
$$

where

${ }^{i} \vec{V}_{A / G},{ }^{i} \vec{V}_{B / G}=$ relative velocity of points $\mathrm{A}$ and B with respect to $G$

$\omega=$ angular velocity of segment

Positive rotational speed of the segment is considered counterclockwise. Having defined the endpoint velocities, their relative motions are constrained by axial and bending springs and dampers. The velocity of center of mass $(G)$ of each segment, while defined in the local coordinate system, must be transformed to the inertial coordinate system in order to apply gravity, and to allow system initialization through a static vertical displacement of a point on the cable, which is then released to create free vibration. The coordinate transformation for velocity of point $G$ is as follows.

$$
{ }^{I} \vec{V}_{G}=\left[\begin{array}{l}
V_{G x} \\
V_{G y}
\end{array}\right]=\left[\begin{array}{cc}
\cos \theta & -\sin \theta \\
\sin \theta & \cos \theta
\end{array}\right]{ }^{i} \vec{V}_{G}
$$

where $\theta=$ angle of rotation of segment $i$.

Also, because the end point velocities of each segment need to be related, both end point velocities should be expressed in the same frame, in this case the local coordinate of the segment to the right. Eq. (7) represents the coordinate transformation relation between two connected sections.

$$
{ }^{i+1}\left[\begin{array}{c}
V_{A x} \\
V_{A y}
\end{array}\right]=\left[\begin{array}{cc}
\cos \left(\theta_{i+1}-\theta_{i}\right) & \sin \left(\theta_{i+1}-\theta_{i}\right) \\
-\sin \left(\theta_{i+1}-\theta_{i}\right) & \cos \left(\theta_{i+1}-\theta_{i}\right)
\end{array}\right]^{i}\left[\begin{array}{c}
V_{A x} \\
V_{A y}
\end{array}\right]
$$

Eq. (8) represents Newton's Law for the segment in local coordinates.

$$
{ }^{i} \sum \vec{F}=\frac{d}{d t}{ }^{i} \vec{P}={ }^{i}\left[\begin{array}{l}
m \dot{v}_{x} \\
m \dot{v}_{y}
\end{array}\right]+\vec{\omega} \times\left[\begin{array}{l}
m v_{x} \\
m v_{y}
\end{array}\right]=\left[\begin{array}{l}
m \dot{v}_{x} \\
m \dot{v}_{y}
\end{array}\right]+\left[\begin{array}{c}
-m \omega v_{y} \\
m \omega v_{x}
\end{array}\right]
$$

The first term on the right-hand side is modeled with generalized inertias in the bond graph, and the second term using modulated gyrators.

\section{Bond Graph Model}

The bond graph model of the cable consists of 30 segments, which is enough to produce the first few frequencies with reasonable accuracy. Each segment is connected to the next segment with a connection sub-model and each connection sub -model contains the compliance and damping bond graph elements. Figure 2 shows the bond graph model of a segment of the cable. A bond graph 1-junction represents a generalized Kirchoff loop law, with all bonded elements having the same flow, and efforts algebraically summing to zero. A bond graph 0 -junction represents a generalized node law, with all bonded elements having the same effort. The 0 -junction also sums velocities of bonded elements to zero, making it the element used to enforce velocity constraints. As can be seen from Figure 2 , there are two modulated transformers (MTF), one for each row of Eq. (6), two MTF's for defining Eq'ns (4) and (5) and one modulated gyrator (MGY) for defining the Euler's Equations inner product terms of Eq. (8). The power bonds (with half-arrows) contain both force and velocity information. In Figure 2 sample junction equations are given. The 1-junction representing Newton's Law in the $x$ direction sums forces, including constraint forces from adjoining elements that are on the same bonds that communicate velocity of points A and B to adjoining elements. Left superscripts indicate the local $(i)$ or inertial (0) frame. Figure 3 shows the bond graph of one connection sub-model between two adjoining segments. As can be seen in Figure 3, there are four MTF's for defining coordinate transformation Eq. (7). The top row of the bond graph represents the velocities and elements in the $x$ and $y$ directions and the most bottom row represents the bending compliance.

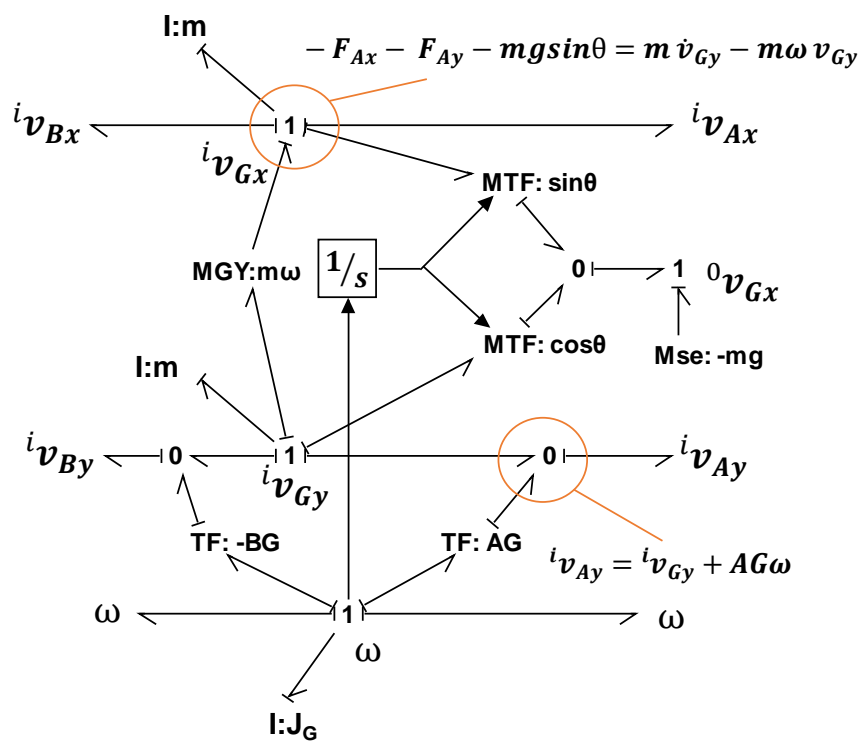

Figure 2. Bond Graph Model of a Segment of the Cable 
TABle 1. MATERIAL PROPERTIES OF CABLE

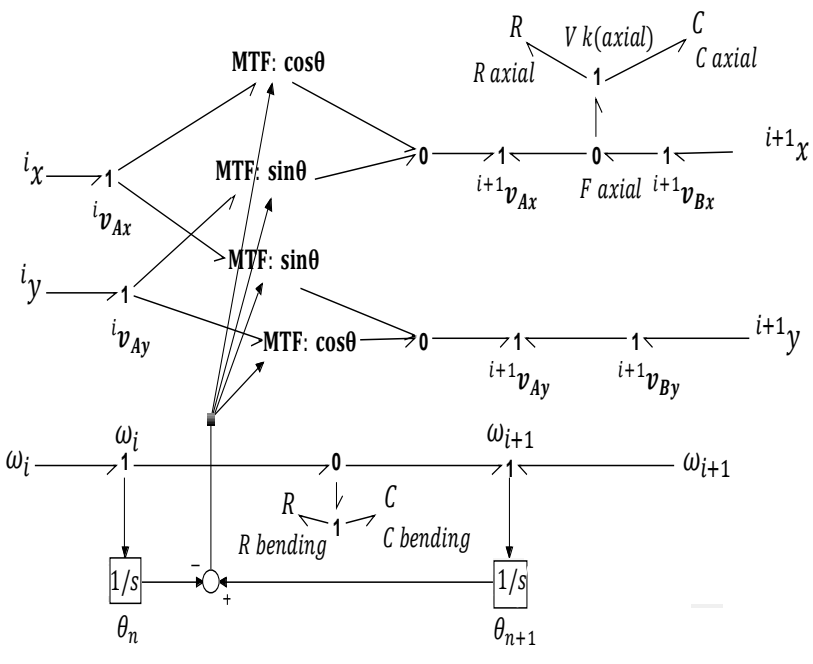

Figure 3. Bond graph model of complaint connection between consequent segments

In Figure 3, the 0-junction $F_{\text {axial }}$ defines the velocity $v_{k \text { (axial) }}$ as the difference between $v_{B x}$ on segment $i+1$ and $v_{A x}$ on segment $i$ defined in the $i+1$ coordinate system. The 1junction equates the velocities of the parallel axial spring and damper. The bending spring/damper velocity is defined similarly. The block diagram elements define the relative angle between segments for the velocity transformation.

Point B of the left-most segment and Point A of the rightmost segment are attached to the wall and therefore have zero velocity. Thus, two zero flow sources are used at each point in the $x$ and $y$ directions. In order to create the desired initial tension in the cable, the model is given zero initial conditions and then stretched until the desired tension is achieved. In this paper, four values of tension are considered for the analysis. For applying the stretching displacement to the right end of the cable in bond graph model, a flow source was temporarily applied to the 1-junction associated with the velocity of the right end of the cable in local $x$ direction. After creating the desired tension, the zero flow source was reinstated at the right end. The cable was then excited, with results of the bond graph model compared to experimental results.

\section{EXPERIMENTAL VIBRATION TESTING OF THE CABLE}

\section{A. Cable Bending Stiffness Measurement}

The material properties of the conductor used for testing are tabulated in Table 1 . The bending stiffness $(E I)$ of the cable should be measured as it is stranded cable and its bending stiffness is not equal to the bending stiffness of solid cable with the same diameter [6]. Figure. 4 shows the setup used for measuring the bending stiffness $(E I)$ and consequently area moment of inertia $(I)$ of the cable.

\begin{tabular}{|c|c|c|c|c|}
\hline Material & $\begin{array}{c}\text { Length } \\
{[\mathrm{m}]}\end{array}$ & $\begin{array}{c}\text { Density } \\
{\left[\mathrm{kg} / \mathrm{m}^{3}\right]}\end{array}$ & $E[\mathrm{GPa}]$ & $\begin{array}{c}\text { Area } \\
{\left[\mathrm{m}^{2}\right]}\end{array}$ \\
\hline $\begin{array}{c}\text { Stainless } \\
\text { Steel 7× } \\
7 \text { wires }\end{array}$ & 3.6 & 9537 & 195 & $2.78 \times 10^{-5}$ \\
\hline
\end{tabular}

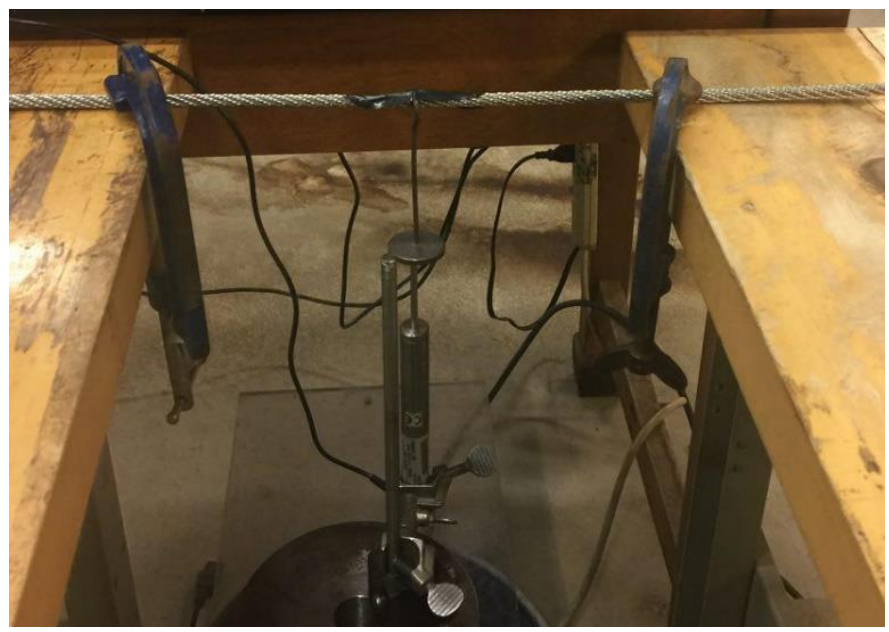

Figure 4. Set up for Bending Stiffness Measurement

A section of the cable is clamped between two ends and a fixedfixed configuration is made. Various weights are hung in the middle of the cable section and a LDVT is used to measure the deflection of the cable at midspan. By using standard forcedeflection formulae for a fixed-fixed beam with force applied at midspan, the bending stiffness of the cable is obtained [13]. It should be noted that the value of measured $E I$ from bending test is between theoretical minimum and maximum bending stiffness bounds for the stranded cables. $E I_{\min }$ corresponds to when all the layers of the cable slip on each other and $E I_{\max }$ is for all the wires are bonded and make a solid cable. The details of the theoretical bounds for bending stiffness can be found in [6]. Bending of the cable in a cantilever configuration was also performed with different lengths of the cable and the results were the same, which shows that the bending stiffness of the cable is not sensitive to length, over the range of segment lengths considered in this work. A measured constant value for $E I$ is directly used in the bond graph model in numerical analyses. Table 2 presents the value of $E I$ for three different lengths of cable sections.

TABLE 2. VALUE OF MEASURED EI WITH DIFFERENT LENGTHS

\begin{tabular}{|c|c|}
\hline Length $(\mathrm{mm})$ & $E I\left(\mathrm{Nm}^{2}\right)$ \\
\hline 205 & 0.555 \\
\hline 364 & 0.444 \\
\hline 400 & 0.547 \\
\hline
\end{tabular}




\section{B. Experimental Modal Testing}

Figure 5 shows the set up used for modal impact testing of the cable.

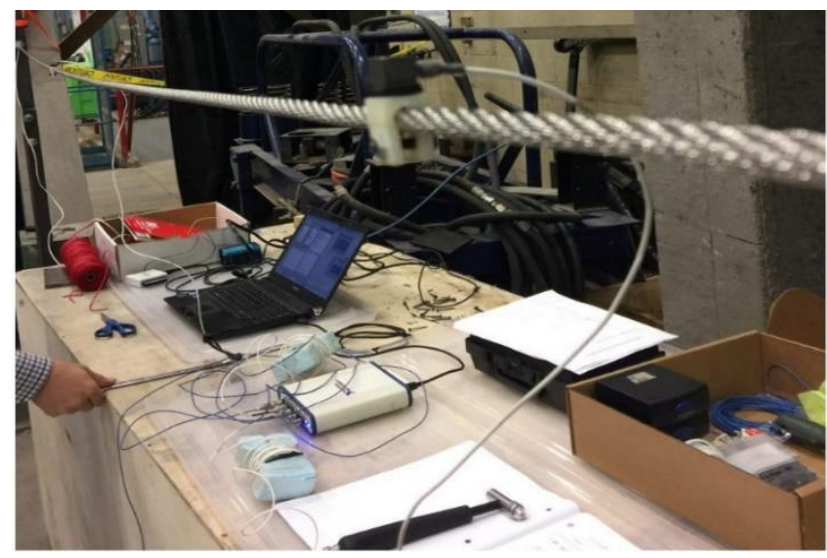

Figure 5. Cable Vibration Test Set up

Figure 6 presents a schematic illustrating two set-ups used for testing the cable. Two pin support structures hold the cable. The right end of the cable is attached to a threaded rod to adjust the static tension of the cable. A load cell is at the left end of the cable to measure the longitudinal tension of the cable. The load cell is directly inline with the cable to measure the longitudinal tension of the cable. The rectangular solid shapes and the black arrows represent respectively the position of the accelerometers and the hammer hits along the cable.

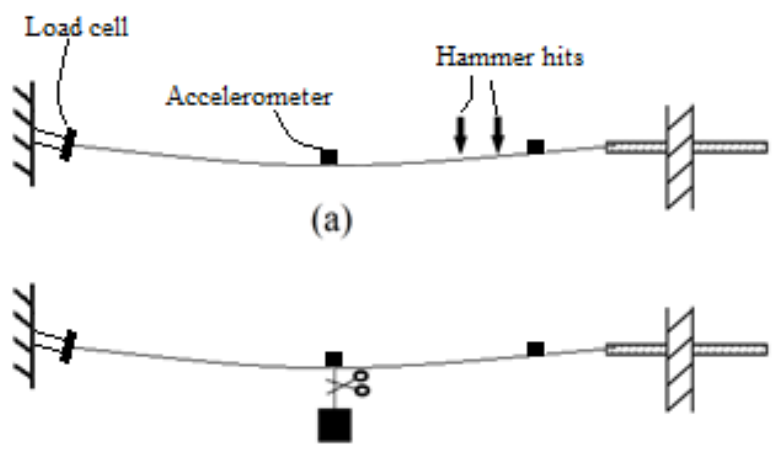

(b)

Figure 6. Schematic view of cable vibration testing

Modal tests are performed with a Bruel \& Kjaer 8205-002 impact hammer, 4507 B 004 70-g accelerometers, and a National Instruments NI USB-4432 power supply and signal conditioner. Cable tension is measured with a load cell. Also, the software ModalView [14] is used to analyze the data. All modal tests averaged 5 hits.

Figure 6 (a) illustrates the set up used to perform hammer modal testing over the cable, experiment "A". With accelerometers positioned at 0.3 and $1.8 \mathrm{~m}$ from the right side, the measurements taken hitting the hammer in two different positions, at $1 / 4$ and $1 / 5$ of the cable span. The modal test was performed with different tensions which are tabulated in Results section.
Another experiment, " $\mathrm{B}$ ", is performed as illustrated by Figure 6 (b). The cable is supported the same way as before, however now there is a mass attached to the middle of the cable through a string. Cutting the string creates a force excitation that can be replicated in simulation.

\section{RESULTS AND DISCUSSIONS}

The Frequency Domain Toolbox in the 20sim ${ }^{\circledR}$ bond graph simulation environment numerically generates a system transfer function from which eigenvalues are calculated. Table 3 presents the experimental and numerical results of the natural frequencies and damping ratios of the tensioned cable. In the simulation, an impulse force is applied at the quarter of the cable span which is in the 7 th cable segment and the time response is obtained at the midspan of the cable. The result of experiment (b) in Figure 6 and corresponding simulation results are illustrated in Figure 7 and Figure 8. The measured and simulated acceleration time response of the midspan can be compared from these figures. Figures 7-8 show accelerations of similar magnitude. Discrepancies are primarily attributed to inaccurate high-frequency modes inherent in a lumped-segment representation. Figure 9 presents the stabilization diagram obtained from ModalView to extract the natural frequencies of the cable with $350 \mathrm{~N}$ tension. The peaks in the stabilization diagram correspond to the natural frequencies of the cable.

\begin{tabular}{|c|c|c|c|c|}
\hline \multirow{2}{*}{$\begin{array}{c}\text { Tension } \\
(\mathrm{N})\end{array}$} & \multicolumn{2}{|c|}{ Modal Testing } & \multicolumn{2}{|c|}{ Simulation } \\
\hline & $\begin{array}{l}\text { Freq } \\
(\mathrm{Hz})\end{array}$ & $\begin{array}{c}\text { Damp } \\
(\%)\end{array}$ & $\begin{array}{c}\text { Freq } \\
(\mathrm{Hz})\end{array}$ & $\begin{array}{c}\text { Damp } \\
(\%)\end{array}$ \\
\hline \multirow[t]{3}{*}{91.4} & 2.37 & 4.025 & 2.41 & 1.612 \\
\hline & 4.52 & 16.4 & 4.77 & 11.511 \\
\hline & 6.88 & 3.880 & - & - \\
\hline \multirow[t]{3}{*}{140.6} & 4.53 & 0.645 & 4.21 & 5.672 \\
\hline & 6.53 & 0.939 & - & - \\
\hline & 10.15 & 1.18 & 13.03 & 2.014 \\
\hline \multirow[t]{3}{*}{230.8} & 4.96 & 1.074 & 5.19 & 4.223 \\
\hline & 8.19 & 0.699 & 8.32 & 1.770 \\
\hline & 12.39 & 0.900 & 13.31 & 54.881 \\
\hline \multirow[t]{3}{*}{300.7} & 5.14 & 0.913 & 5.11 & 32.85 \\
\hline & 9.46 & 0.357 & 9.19 & 91.908 \\
\hline & 14.22 & 0.436 & 14.8 & 2.741 \\
\hline \multirow[t]{3}{*}{350} & 5.35 & 0.878 & 5.02 & 1.918 \\
\hline & 10.18 & 0.258 & 10.34 & - \\
\hline & 15.27 & 0.343 & - & - \\
\hline \multirow[t]{3}{*}{400} & 5.58 & 0.807 & 5.07 & 17.997 \\
\hline & 10.87 & 0.209 & 10.3 & 5.509 \\
\hline & 16.27 & 0.487 & 15.23 & 0.778 \\
\hline
\end{tabular}




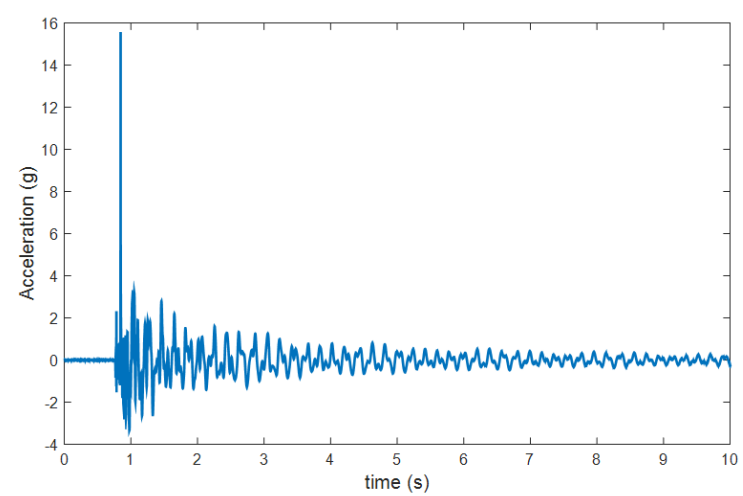

Figure 7. Acceleration time series of the midspan of the cable- Experimental result

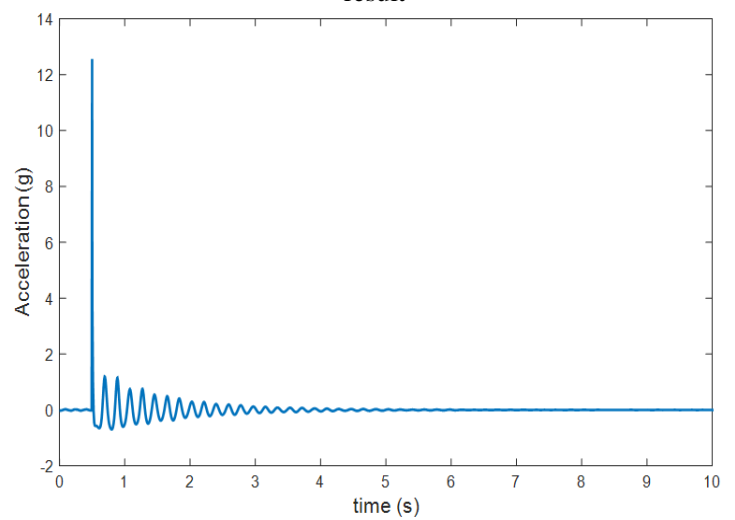

Figure 8. Acceleration time series of the midspan of the cable- Simulation

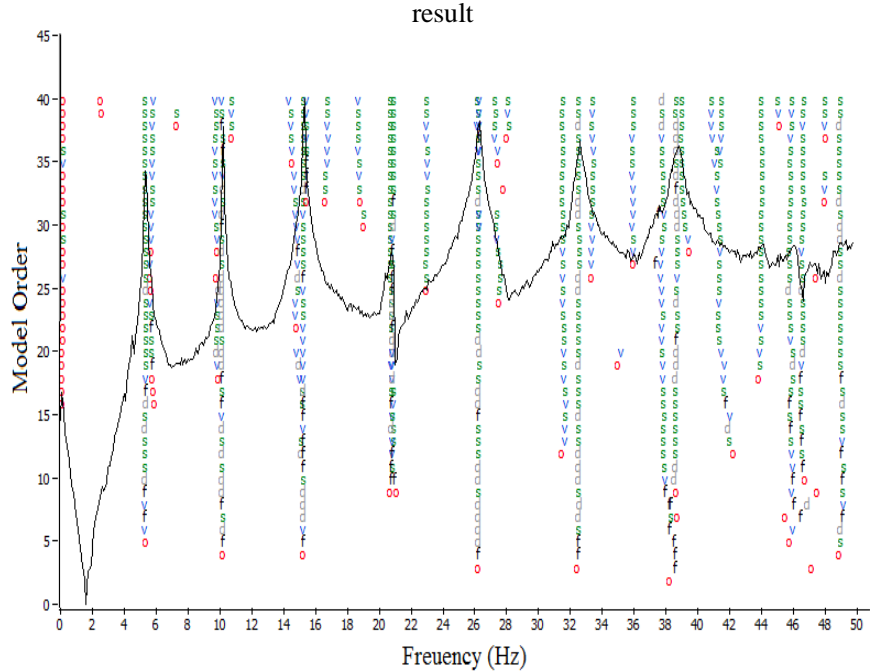

Figure 9. Experimental stabilization diagram

\section{CONCLUSIONS AND FutURE WORK}

A bond graph lumped segment model was developed to obtain the modal data of a conductor. The natural frequencies and damping ratios from modal testing of the physical setup were compared to those from simulation, along with time responses. The overall system frequencies were predicted with the simulation model for the range investigated. Discrepancies in damping ratios, and in time response, are attributed to the use of simple linear viscous damper elements. Developing and tuning a more accurate damping model remains an open research problem. In addition, as the overall goal of the research is to capture the effects of cable on dynamics of poles, cable numerical models should be combined with the pole models to investigate the effects of cables in a line of pole-cables in the future work.

\section{REFERENCES}

[1] O. Barry, J. Zu, D. Oguamanam, Analytical and experimental investigation of overhead transmission line vibration, Journal of Vibration and Control 21(14) (2015) pp. 2825-2837.

[2] G. Ricciardi, F. Saitta, A continuous vibration analysis model for cables with sag and bending stiffness, Engineering Structures 30 (2008) pp. 1459-1472.

[3] N. Barbieria, M. Kelly, T. Calado, M. Josém, Mannalac, K.F.d. Lima, G.d.S.A.V. Barbieria, Dynamical analysis of various transmission line cables Procedia Engineering 199 (2017) pp. 516-521.

[4] H. Li, W. Shi, G. Wang, L. Jia, Simplified models and experimental verification for coupled transmission tower-line system to seismic excitations, Journal of Sound and Vibration 286 (2005) pp. 569-585.

[5] M.L. Q. Wang, H.N. Li, Aseismic calculation of transmission tower-conductor system, Earthquake Engineering and Engineering Vibration 9 (1989) pp. 73-87.

[6] K.O. Papailiou, On the bending stiffness of transmission line conductors, IEEE Transactions on Power Delivery 12 (1997) pp. 1576-1588.

[7] K. Spak, G. Agnes, D. Inman, Cable Modeling and Internal Damping Developments, Applied Mechanics Reviews 65 (2013).

[8] Kaitlin S. Spak, G.S. Agnes, D.J. Inman, Modeling vibration response and damping of cables and cabled structures, Journal of Sound and Vibration 336 (2015) pp. 240-256.

[9] K.S. Spak, G.S. Agnes, D.J. Inman, Toward Modeling of Cable-Harnessed Structures: Cable Damping Experiments, 54th AIAA/ASME/ASCE/AHS/ASC Structures, Structural Dynamics, and Materials Conference, Boston, Massachusetts, 2013.

[10] P.F. Pinto, D.G. Rideout, Development and validation of an in-situ utility pole simulation model for virtual modal testing, Proceedings of the ASME 2016 IMECE International Mechanical Engineering Congress \& Exposition IMECE2016, Phoenix, Arizona, USA, 2016.

[11] D.C. Karnopp, D.L. Margolis, R.C. Rosenberg, System Dynamics: Modeling, Simulation, and Control of Mechatronic Systems, Fifth ed., John Wiley and Sons, 2012.

[12] L. Meirovitch, Fundamentals of Vibrations, McGraw Hill, 2000.

[13] R.C. Hibbeler, Mechanics of materials, Pearson, 2011.

[14] ModalVIEW, ABSignal b.v., 2012. 